\title{
ECMO - Challenges, Strategies and Preparation from Spain
}

\author{
Enrique Pérez de la Sota ${ }^{1}$ and Andrea Eixerés-Esteve ${ }^{1}$ \\ ${ }^{1}$ Hospital Universitario 12 de Octubre
}

July 30, 2020

\begin{abstract}
Providing complex therapies such as ECMO during outbreaks of infectious diseases has singular challenges. The impact of the SARS-CoV-2 pandemic has implied a mentality change by force of circumstances, and cardiac surgery has not been stranger to this trend. The need to treat critically ill patients with an unknown evolution has compelled cardiovascular surgeons to decide whether or not to implant an ECMO system, despite the limited scientific evidence available in the context of COVID-19. To add some confusion, doubts were raised about its potential deleterious outcome in COVID-19 patients, due to its effect on lymphocyte counts and interleukin- 6 concentrations. The care of the critically ill patient in a moment of national emergency in Spain took precedence over those possible formal doubts. The Spanish perspective on Ventricular Assist Devices during and after the COVID-19 pandemic, focused on ECMO as a particular case of mechanical circulatory support, is presented. We address both the challenges posed by the pandemic and the organizational model established in Spain; changes in ECMO therapy and some lessons learned for the next outbreaks are also described. It is not about reinventing the wheel in each country; it is enough to learn from experience and take advantage of the knowledge generated by those who have already gone through similar situations in our environment.
\end{abstract}

ECMO - CHALLENGES, STRATEGIES AND PREPARATION FROM SPAIN

\section{AUTHORS}

Enrique Pérez de la Sota MD, Andrea Eixerés-Esteve MD

INSTITUTION AND AFFILIATIONS

Cardiac Surgery Department, 12 de Octubre University Hospital, Madrid, Spain.

SHORT RUNNING TITLE: ECMO\&COVID in Spain

ADDRESS FOR CORRESPONDENCE

Dr. Enrique Pérez de la Sota

Cardiac Surgery Department - 12 de Octubre University Hospital

Avenida de Córdoba s/n. 28041 Madrid - SPAIN

Email: epsota@gmail.com

Phone number: +34689443265

Fax number: +34 914740658

FUNDING SOURCES : None

INTRODUCTION 
On March 14, 2020, the government of Spain declared a State of Alarm across the country with a decree that imposed extremely strict conditions and, in practice, instituted a national lockdown. All healthcare-related matters fell under the direct orders of the Minister of Health, although certain possibilities for managing the corresponding health services were granted to the regional and local public administrations. In practice, all material and human health resources were devoted exclusively to the fight against the pandemic, and all scheduled surgical activities were canceled.

The Spanish Society of Cardiovascular and Endovascular Surgery (SECCE) reacted expeditiously to the new situation created by the pandemic and established a series of recommendations ${ }^{1}$ to ensure care in different healthcare dimensions: emergencies, surgical prioritization of preferential patients, safe delay of elective patients, and adaptation of ECMO programs in the context of COVID-19.

\section{CHALLENGES}

\section{Ventricular Assist Devices - Heart Transplant}

Pre-transplant circulatory support in Spain has traditionally been done with short-term devices that enabled effective and sufficient support until the arrival of the organ in a matter of days, since the donation system by the Transplant National Organization (the "Spanish Model") has achieved short waiting times. The tradeoff is that long-term circulatory support has had a slow development and a reduced experience in Spain in comparison with the surrounding countries, amounting to a total of 40-50 implants per year for all indications in recent years.

In this scenario, the impact of the coronavirus pandemic on ventricular assist devices has been minimal. Excluding ECMO support to COVID-19 patients, emergency implants have only been performed in situations of cardiogenic shock and as urgent pre-transplant support, and even in these cases, the devices used have been short-term ones (Impella ${ }^{\circledR}$, ECMO). Elective heart transplants were suspended in most of the Spanish groups for two months, so non-urgent patients, assisted with long-term devices, remained on the waiting list. In the post-COVID era, we should not expect major changes in both device indications and implantation strategy.

\section{ECMO Therapy}

\section{Background :}

The need to treat critical COVID-19 patients has forced cardiovascular surgeons to decide whether or not to implant an ECMO system. As there is only scant and unsubstantiated evidence available hitherto, with only minor experiments conducted, limited to a few published cases from China ${ }^{2-4}$ and some literature reviews, such as that of Savarimuthu et al.$^{5}$, the benefits of ECMO system implantation are not clear.

To add some confusion to the issue, doubts were raised about the possible deleterious effect of ECMO in this disease. Patients who die of COVID-19 have significantly lower lymphocyte counts and significantly higher interleukin-6 (IL-6) levels than survivors. It is a well-known fact, that during ECMO support there are substantial decreases in the number and function of some lymphocyte populations, as well as consistently high IL-6 levels, which are inversely correlated with survival ${ }^{6}$. Elevated concentrations of IL-6 in the lungs, induced by starting ECMO, have also been shown to be associated with parenchymal damage in porcine models of veno-venous (VV) $\mathrm{ECMO}^{7}$.

In this context, it would be considered prudent to evaluate the immune status of ECMO candidate patients and seriously consider monitoring lymphocyte count and IL-6 during support, while the pressure of care and the number of critical patients that cannot be ventilated conventionally grew uncontrollably.

Official regulation of the Government of Spain:

On February 28, the Ministry of Health published a technical document ${ }^{8}$ entitled "Clinical management of COVID-19: intensive care units", with the participation of numerous scientific societies and medical organizations and with successive updates, in line with the evolution of the health crisis. 
The April 27 version reviews and updates the section on ECMO and advises starting care in patients with Acute Respiratory Distress Syndrome (ARDS) who, despite the outlined protocols having been followed, have persistent severe respiratory failure meeting the criteria indicated by that document ${ }^{8}$. It establishes that support must be offered in centers with proven experience, with well-structured programs, and with an annual minimum of cases $>15$. As stated in the document, "if the guidelines of this protocol are followed, both in relation to the measures to be applied before assistance, and in relation to the indications and contraindications of their initiation, it is calculated that the need for ECMO will range from 4 to $7 \%$ ".

SECCE's corporate recommendations ${ }^{1}$ :

The SECCE established that cardiovascular surgery services, in conjunction with those of Intensive Care Medicine, could offer specialized last-level care through the use of temporary circulatory mechanical support systems (veno-venous and veno-arterial ECMO) in COVID-19 patients with severe ARDS or cardiomyopathy refractory to treatment. With this official position, the care of the critically ill patient in a moment of national emergency took precedence over possible formal doubts about the suitability of ECMO therapy in this disease $\mathrm{e}^{9,10}$. The recommendations established a series of key points as main lines of action against the pandemic:

- Adapting the ECMO programs of the different hospital centers for their priority use in the pandemic, also guaranteeing adequate health care in the reference area (first- and second-tier hospitals) and without neglecting other non-COVID patients who are possible candidates for the therapy.

- Specifically establishing the ECMO (VA and VV) entry criteria, with some parameters quite similar to those defined somewhat later by the ELSO Guidelines for ECMO in COVID-1911, and the exclusion and contraindication criteria for the ECMO therapy in COVID-19 patients (Figure 1, in Spanish).

- Limiting the number of professionals participating in the procedure to the minimum necessary; the number of professionals involved at each moment of the implantation, the inflow and outflow of each one of them from the ICU room in which the implantation was made, as well as the level of protection required for each person was precisely detailed, depending on their specific role in each phase of the process.

- Enrolling the patient in the COVID-19 ECMO Registry that the SECCE created for this purpose.

The different services were organized to offer the assistance required at all times according to the impact of the pandemic, and a map of device use was established, with a progressive restriction of the entry criteria based on the availability of resources and each center's own clinical experience.

Records of ECMO implantation in COVID-19 patients:

EuroECMO-COVID Survey :

This is a direct initiative of the Steering Committee of the European Chapter of the ELSO (Extracorporeal Life Support Organization) in order to obtain information on the use of ECMO in European and Israeli centers in patients with COVID-19. Data collection began on March 15, 2020, and there was a first online publication with 333 patients in late April ${ }^{12}$. As of July 17, and with 1,387 patients collected, the survey reflected 160 ECMO implants in 26 centers for Spain, which placed us in 3rd place in Europe in number of implants, behind France (367 devices) and Germany (165 devices).

Spanish Registry of ECMO Implants in COVID-19:

The foreseeable increase in the use of ECMO devices caused by the very high incidence of cases in our country led to the creation of a Registry of Implants in patients with COVID-19 (coordinator: Dr. Mario Castaño, León, Spain) managed by the SECCE and its Mechanical Circulatory Support Working Group.

It is based on the EuroECMO-COVID Survey and allows direct exportation to it, although the Spanish registry is more ambitious when incorporating evolution variables, analytical-prognostic parameters, and severity markers (D-dimers, procalcitonin, C-reactive protein, ferritin, etc.) collected during the entire support period. Altogether 100 variables are collected in a secure database accessible online. 
The registry was launched in early April and, at the time of writing this manuscript, it is still open and there are no preliminary analyses or provisional data. In the near future the definitive results will be published and that is why here we can only give a partial and incomplete view of the information collected, not in the form of scientific communication or official publication. In the first week of July, the registry collected more than 110 patients from 24 different centers with accumulated experience, logically, in the areas with the highest incidence of cases (Madrid and Catalonia); ECMO VV (90\%) was fundamentally implanted in the femoral-jugular configuration in two thirds of the cases, and in up to $10 \%$ of patients it was changed to a VA configuration or a third cannula was associated (V-VA or VV- A) due to oxygenation problems or the appearance of ventricular failure. In almost $90 \%$ of patients, the indication was ARDS and/or pneumonia (in critical COVID-19 patients, differentiating between the two concepts is difficult due to the similarity in symptoms) and the rest corresponded to myocarditis, heart failure, barotrauma, or an association of several of these.

Today, more than $50 \%$ of patients have been successfully disconnected from ECMO and almost $20 \%$ more are still in support. Of all those who have completed the follow-up, $55 \%$ were discharged from the hospital, although the final data for each patient is not available at the present time, in the expectation of later seeing the evolution of the pandemic and deciding on the date of definitive closing of the registry.

\section{STRATEGIES}

\section{Webinars as an alternative form of training}

The total lack of knowledge about the virus and its effects, the lack of scientific evidence, and the urgent need for direct information on the management of different scenarios in the pandemic have translated into great proactivity towards the creation of forums and activities of an educational nature. Especially in the weeks following the worst moments of the pandemic, it was not rare to receive invitations to seminars, webinars, and other online medical events in which professionals from all over the world participated.

The SECCE was no stranger to this trend; the boost that COVID-19 has given ECMO therapy and its high implantation rate in Spain gave rise to a first event on May 4, the "Virtual Symposium: ECMO in COVID patients", in which the author (EPS) had the honor of participating together with other Spanish colleagues and which was followed by another international event organized by the Spanish Association of Perfusionists and the Latin American Perfusion Association, "COVID-19: New trends in perfusion", with the participation of surgeons (Spain and Germany) and perfusionists (Spain and Switzerland). Both were highly successful in attendance and interactive participation by Spanish and Latin American professionals, from different specialties and fields of work.

As a culmination of the ECMO therapy training initiative, the SECCE has organized the 1st Online Course "ECMO, the success of therapy" (http://sectcv.es/category/formacion/simposio-virtual/) with a global and multidisciplinary approach. Participating teachers include cardiovascular surgeons (adult and pediatric), anesthetists, intensivists, cardiologists, perfusionists, and intensive care nurses. In five modules, the program covers all aspects related to the use of ECMO:

- Module I: Indications and versatility of ECMO (indications, management and cannulation)

- Module II: ECMO, state of the art (physiology, anticoagulation, complications).

- Module III: Thinking out of the box? (Pediatric ECMO, ECMO in heart donation).

- Module IV: New frontiers for ECMO (transplantation, transfers, ethical aspects).

- Module V: Summary (lecture by Dr. Daniel Goldstein - Montefiore Medical Center, NYC - and panel discussion with experts).

This need to acquire knowledge remotely has given a boost to the Society's online training offerings which, although already in existence, have now been reinforced and expanded with new online courses and seminars 
(http://campuscardiovascular.sectcv.es) about other aspects of cardiovascular surgery not directly related to COVID-19.

\section{Redesign of the health system}

The enormous pressure on hospitals due to the COVID-19 pandemic should spark reflection on whether these institutions should be the first resort when people are sick. With adequate means of infection control, certain locations such as outpatient hospitals or hospital rehabilitation centers could be the initial destination for COVID-19 patients who have been sent to hospitals during the first phase of the pandemic. This is already being done in other countries such as the USA, where many service and care provider organizations and some community clinic systems are diversifying in this way ${ }^{13}$.

\section{Reorganization of resources}

Italy was the first European country to be massively hit by the SARS-CoV-2 infection, and for this reason it had to react quickly and effectively to the resulting health chaos, especially in the Lombardy region, which was the most strongly affected at first. This reaction has been exemplary, with a change in the healthcare model introduced almost immediately. On March 8, 2020, the Italian government announced the closure of the affected regions and that same day, the Lombardy regional government reviewed the entire system and introduced new regulations on how to reorganize hospitals according to a "hub \& spoke" model. Four hub centers were created for adult cardiac surgery and one for pediatric surgery, which would handle all emergency cases (150-200 cases/month) and the existing ECMO network remained unchanged ${ }^{14}$.

Due to the special decentralized administrative structure of Spain, with the health competences assigned to regional governments and the hospital organization regulated by the management of each center, the restructuring of cardiac surgery during the pandemic has been similar to that of Italy, but without the radial scheme. Each hospital has operated in a nuclear manner, with the independence to define surgical strategies, relocate ICUs, and organize emergency services. In practice, the action has been quite homogeneous and followed the guidelines set by the SECCE ${ }^{1}$. All elective medical activity (ambulatory and surgical) was canceled, some hospitals were completely transformed into COVID-19 hospitals, exclusive routes were organized for patients with COVID-19 within the hospital, and completely new COVID-19 intensive care units were created in different hospital facilities (operating theaters, postsurgical critical care areas, rehabilitation gyms, etc.).

\section{ECMO networks}

The decision to use ECMO in COVID-19 patients relates not only to the expected benefit of therapy and possible risks, but also to the availability of ECMO supplies and hospital infrastructure, and personal and group experience. These last two points are essential when establishing joint strategies and guaranteeing an adequate organization of personnel, equipment, facilities, and systems. As with any scarce resource in times of high demand, mismatches may appear in these factors, and it is preferable to anticipate with an action plan that develops a sustainable system to ensure quality of care.

In Spain, in the pre-COVID era, there was no uniform organization for ECMO care. In some regions (Valencia, Catalonia, or Eastern Andalusia, for example), there were well-defined programs, with clear action coordinates for referring patients to ECMO centers, and even with availability for remote implants and inter-hospital transport. However, in other areas, each hospital that had the therapy offered it openly to its secondary and regional reference hospitals in an unorganized way and without defined patient flows. The same peripheral hospital could send some cases to its referral hospital for cardiac surgery (and ECMO therapy) and send other cases to any of the tertiary hospitals in its environment, without any administrative interference and by simple decision of the treating doctor.

The arrival of the pandemic, with the sudden increase in the need for intensive care beds and extracorporeal respiratory support, meant that a well-defined referral and transfer system needed to be organized from each regional Health Administration to provide access to the specialized care needs of the patients of all the 
first- and second-tier hospitals. ECMO Reference Hospitals were designated and their satellite hospitals were specified.

This has planted the seed for an ECMO Network system on a hub \& spoke model which, while not yet fully developed in all areas, seems to perfectly fit the needs of the new organization and could be consolidated in the future for all ECMO care nationwide.

\section{Resources:}

Training in ECMO must be ensured for all members of the group to be able to act as reinforcement or replacement in case of illness of the professionals initially assigned to the ECMO pandemic team.

It is mandatory to periodically update the inventory of available machines ready for use and to forecast the needs so as to ensure a constant supply of consumables.

\section{Contact between hospitals:}

The success of ECMO therapy is based, among other factors, on an early implantation when the clinical indication is clear, and this involves not deferring the decision when the previous therapeutic steps have not yielded the expected result. In a pandemic situation, this is especially relevant and therefore there must be frequent communication between the satellite hospital and the ECMO hospital, assessing the evolution of the most seriously ill patients and anticipating situations of sudden deterioration (not infrequent in critically ill COVID-19 patients) in which the ECMO option is possibly already too late. If the speed of progression of the disease from dyspnea to ARDS is rapid or unknown, we recommend early transfer (after tracheal intubation, for example) to an ECMO center, wherever possible.

This fact is considered critical in the technical document of the Ministry of Health, and for this reason it explicitly states that "in the event that the center does not have the technique, transfer to a reference center must be considered" and that "there must be early contact between centers", according to clinical and analytical criteria summarized in Table 1.

Inter-hospital transport:

In our country there are mobile programs for ECMO implantation and inter-hospital transport of assisted patients (La Fe Hospital in Valencia, Bellvitge Hospital in Hospitalet de Llobregat-Barcelona, Virgen de las Nieves Hospital in Granada, among others) that offer appropriate service to their reference area, but they are a minority in Spain and otherwise, there are only isolated experiences with remote implants both in adults and in pediatric patients (Hospital 12 de Octubre in Madrid).

The hasty reorganization of ECMO assistance necessitated by the pandemic is a good starting point to try to expand such initiatives and establish a well-organized network of remote ECMO assistance with all its possibilities. It seems clear that high-volume ECMO centers, particularly those serving as a regional benchmark, should establish and coordinate mobile ECMO teams available 24 hours a day, 7 days a week, and made up of personnel trained and experienced in transporting critically ill patients and inserting cannulas.

Among the basic principles on which the constitution of these mobile ECMO teams should be based, in the context of a pandemic, we can point out ${ }^{15}$ :

- Design eligibility criteria for transfer between hospitals and share them with the satellite centers.

- Ensure effective communication and coordination at all times to shorten response times or to explain to the responsible physicians the refusal to transfer a patient.

- Identify and promptly address the rate-limiting steps of the process.

- Strict adherence to infection control protocols during patient transport, with immediate disinfection of the entire circuit and transport vehicles, to prevent and reduce the risk of cross contamination.

\section{PERSPECTIVES AND PREPARATION}

\section{Ethical Issues}


Not only should the planning for the management of a pandemic focus on trying to reduce the spread of the virus or on treating the sick, but also on providing ethical considerations that allow establishing certain $\operatorname{limits}^{16}$. In this sense, and in a situation like the one experienced in Spain beginning in March 2020, it is totally necessary to ask certain questions such as:

- Should critical care resources be allocated according to the needs caused by the pandemic or according to specific criteria independent of the pandemic situation?

- Is it ethically permissible to use exclusion criteria for scarce resources such as ECMO based on concepts such as objectivity, equity, or transparency of the system?

- Is it ethically permissible to withdraw care from a patient in a critical care unit in order to reallocate resources to another person with a greater chance of benefiting from those resources?

- If new resource allocation standards for critical care and/or ECMO therapy are implemented during the crisis, should clinicians who do not support such implementation be relocated to non-clinical roles, or should it be accepted as a conscience clause that they be released from their obligation to participate in clinical care?

The aforementioned issues have sparked enormous controversy in Spain, not only on a medical level but also among public opinion and the media, and the contest has finally moved to the political scene. The controversy has focused on patients older than 75-80 years, many of whom were not transferred from their retirement homes or residences to hospitals or once admitted to hospital centers, did not have access to critical care beds or ECMO support. The controversy is not over, and it even seems inevitable that it will have judicial ramifications.

The other major ethical concern that involves us, as cardiac surgeons, is the need to continue our surgeries in the event of a pandemic, as a shortage of beds and respirators is expected in the Intensive Care units. In this scenario, it seems reasonable to cancel elective surgeries, but not urgent ones, since the decision to reserve a bed for a critically ill COVID-19 patient and not offer cardiac surgery to a potentially more recoverable patient is a truly conflicting, complicated decision. In the COVID-19 era, the "Heart Team" perhaps becomes more essential and can serve to face certain indications with a more open mind or a more groundbreaking approach that facilitates controversial decision-making by a team and not by one individual.

Focusing on ethical considerations regarding ECMO support during the pandemic, we believe it is essential to keep at least the following points in mind:

- Define and maintain predetermined consensus criteria for the allocation of ECMO in case of a possible shortage.

- Regularly reevaluate all aspects of a patient's treatment plan, including the need to continue or end ECMO therapy.

- Invoke distributive justice only in circumstances where the shortage of devices prevents providing care to every patient.

- In ethically compromising scenarios, it is advisable to seek the opinion of the hospital's ethical and/or medicolegal committees ${ }^{15}$.

\section{Protecting healthcare workers}

The health crisis has made visible the need to duly appreciate the people who work caring for patients and to enable them to carry out their work in complete safety, an aspect that has become glaringly evident in recent months. Spain has achieved the dubious honor of being one of the foremost countries in the world in terms of the number of health professionals infected, with a total of 52,575 by June 25 , and a total of 63 deceased by June 5 (official information from the Ministry of Health). At that time, Spain accumulated a total of 250,545 cases of coronavirus confirmed by PCR, which means that $21 \%$ of the infections corresponds to health personnel (hospitals, health centers and socio-sanitary centers - nursing homes, groups in dependency situations).

In preparation for the more than foreseeable new outbreaks in the coming months, we already have specific 
regulations and self-protection guidelines for ECMO implantation procedures, emergency cardiac surgery, and airway manipulation, thanks to the guidelines published by both professional (SECCE) ${ }^{1}$ and government organizations (Ministry of Health) ${ }^{8}$. When operating with positive COVID-19 patients or patients with unknown infectious status, it is mandatory to use the highest level of personal protection equipment (PPE) available and to follow the standardized procedures (equipping and removing PPE in a sterile environment) developed locally in each center, within the training and education programs for the protection of health workers.

\section{Lessons learned}

In the post-COVID era, the forced incorporation of uncertainty in our lives implies the need to make decisions day by day based on information that we lack, or that is not conclusive enough to make a good decision. We will not be able to depend on certainties - we must be open to learning through personal effort and by strengthening cooperative work.

The opportunity to share experiences with colleagues and experts from other countries and institutions has been very enriching and has enabled, on many occasions, the basis for decision-making to have been the experience of others. This was not applicable for Spain due to the government's lack of foresight, since the correct decisions were not made on time with the information from Italy. In contrast, the Spanish experience has been very useful for our Latin American colleagues, to whose countries the pandemic has come somewhat later and who have been able to anticipate and foresee situations based on what was reported by Spanish colleagues in various joint online forums on ECMO therapy that have unfolded these past months. It is not about reinventing the wheel in each country; it is enough to learn from experience and take advantage of the knowledge generated by those who have already gone through similar situations in our environment.

\section{AUTHOR'S CONTRIBUTIONS}

EPS: concept and design, drafting article, revision and approval of the submitted and final versions of the article. AE-E: concept, critical revision of article, approval of the submitted version.

\section{CONFLICT OF INTERESTS}

The authors declare that there is no conflict of interests

\section{ORCID}

Enrique Pérez-de la Sota http://orcid.org/0000-0002-3816-9739

Andrea Eixerés-Esteve https://orcid.org/0000-0003-2995-7503

\section{REFERENCES}

1. Garrido Jiménez JM, Barquero Aroca JM, Valderrama Marcos JF, et al. Spanish Society of Cardiovascular and Endovascular Surgery recommendations for the COVID-19 pandemic. Cir Cardiov 2020; 27:86-92.https://doi.org/10.1016/j.circv.2020.04.002.

2. Li X, Guo Z, Li B, et al. Extracorporeal Membrane Oxygenation for Coronavirus Disease 2019 in Shanghai, China. ASAIO J . 2020; 66(5):475-481.

3. Zeng Y, Cai Z, Xianyu Y, Yang BX, Song T, Yan Q. Prognosis When Using Extracorporeal Membrane Oxygenation (ECMO) for Critically Ill COVID-19 Patients in China: A Retrospective Case Series. Crit Care 2020; 24(1):148.

4. Yang X, Yu Y, Xu J, et al. Clinical course and outcomes of critically ill patients with SARS-CoV-2 pneumonia in Wuhan, China: a single-centered, retrospective, observational study. Lancet Respir Med . 2020; 8(5):475-481.

5. Savarimuthu S, BinSaeid J, Harky A. The Role of ECMO in COVID-19: Can It Provide Rescue Therapy in Those Who Are Critically Ill? J Card Surg . 2020; 35(6):1298-1301.

6. Henry BM. COVID-19, ECMO, and lymphopenia: A word of caution.Lancet Respir Med. 2020; 8(4): e24.https://doi.org/10.1016/S2213-2600(20)30119-3. 
7. Shi J, Chen Q, Yu W, et al. Continuous renal replacement therapy reduces the systemic and pulmonary inflammation induced by venovenous extracorporeal membrane oxygenation in a porcine model. Artif Organs 2014; 38: 215-23.

8. Ministerio de Sanidad (Gobierno de España). Documento técnico: Manejo clínico del COVID-19: unidades de cuidados intensivos.https://www.mscbs.gob.es/profesionales/saludPublica/ccayes/alertasActual/nCovChina/documentos/Protocolo_manejo_clinico_uci_COVID-19.pdf. Last accessed July 17, 2020.

9. Hong X, Xiong J, Feng Z, Shi Y. Extracorporeal membrane oxygenation (ECMO): does it have a role in the treatment of severe COVID-19? Int $J$ Infect Dis. 2020; 94:7880.https://doi.org/10.1016/j.ijid.2020.03.058.

10. MacLaren G, Fisher D, Brodie D. Preparing for the most critically ill patients with COVID-19: the potential role of extracorporeal membrane oxygenation. JAMA 2020; 323:1245-6.

11. Bartlett RH, Ogino MT, Brodie D, et al. Initial ELSO Guidance Document: ECMO for COVID-19 patients with severe cardiopulmonary failure. ASAIO J. 2020; 66(5):472-474.

12. Marullo AG, Cavaretta E, Biondi-Zoccai G, et al. Extracorporeal membrane oxygenation for critically ill patients with coronavirus-associated disease 2019: an updated perspective of the European experience. Minerva Cardioangiologica 2020 Apr 27.https://www.minervamedica.it/en/journals/minervacardioangiologica/article.php?cod=R05Y9999N00A20042701. Last accessed June 29, 2020.

13. Butler SM. After COVID-19: Thinking Differently About Running the Health Care System. JAMA 2020; 323(24): 2450-2451.https://doi.org/10.1001/jama.2020.8484.

14. Belluschi I, De Bonis M, Alfieri O, et al. First reorganization in Europe of a regional cardiac surgery system to deal with the coronavirus-2019 pandemic. Eur J Cardiothorac Surg . 2020; 58(1): 25-29.

15. Ramanathan K, Antognini D, Combes A, et al. Planning and Provision of ECMO Services for Severe ARDS During the COVID-19 Pandemic and Other Outbreaks of Emerging Infectious Diseases. Lancet Respir Med.2020; 8(5):518-526.

16. Biddison LD, Berkowitz KA, Courtney B, et al. Ethical considerations care of the critically ill and injured during pandemics and disasters: CHEST consensus statement. Chest 2014; 146: e145S-55S.

\section{FIGURE LEGENDS}

Figure 1: Indication, contraindication and exclusion criteria for ECMO in COVID-19 patients.

\section{Hosted file}

Table 1 ECMO Spain JoCS.docx available at https://authorea.com/users/347498/articles/473160ecmo-challenges-strategies-and-preparation-from-spain 


Doble check de intensivista + cirujano
Autorización final de Jefe de Servicio

